ORIGINAL ARTICLE

\title{
Galanin and galanin receptor type 1 suppress proliferation in squamous carcinoma cells: activation of the extracellular signal regulated kinase pathway and induction of cyclin-dependent kinase inhibitors
}

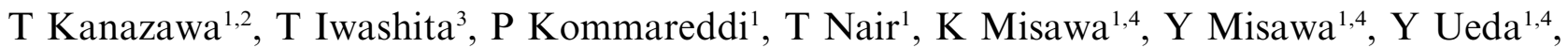 \\ T Tono ${ }^{2}$ and TE Carey ${ }^{1,5}$
}

${ }^{1}$ Laboratory of Head and Neck Cancer Biology, Department of Otolaryngology/Head and Neck Surgery, The University of Michigan, Ann Arbor, MI, USA; ${ }^{2}$ Department of Otolaryngology, Head and Neck Surgery, University of Ryukyus, Okinawa, Japan; ${ }^{3}$ Departments of Internal Medicine and Cell and Developmental Biology, The University of Michigan, Ann Arbor, MI, USA; ${ }^{4}$ Department of Otolaryngology, Hamamatsu University School of Medicine, Hamamatsu, Japan and ${ }^{5}$ Department of Pharmacology, University of Michigan, Ann Arbor, MI, USA

Galanin receptor $1(G A L R 1)$ maps to a common region of 18q loss in head and neck squamous cell carcinomas and is frequently inactivated by methylation. To investigate effects of GALR1 and its signaling pathways, we stably expressed hemaglutinin-tagged GALR1 in a human oral carcinoma cell line (UM-SCC-1-GALR1) that expresses no endogenous GALR1. In transfected cells, galanin induced activation of the extracellular-regulated protein kinase-1/2 (ERK1/2) and suppressed proliferation. Galanin stimulation mediated decreased expression of cyclin D1 and increased expression of the cyclin-dependent kinase inhibitors (CKI), p27 $^{\mathrm{Kip} 1}$ and $\mathrm{p57}^{\mathrm{Kip2}}$. Pretreatment with the ERK1/2-specific inhibitor U0126 prevented these galanininduced effects. Phosphatidylinositol 3-kinase (PI3K) pathway activation did not differ in UM-SCC-1-GALR1 and UM-SCC-1-mock cells after galanin treatment. Pertussis toxin and LY294002 inhibition demonstrated that galanin and GALR1 induce ERK1/2 activation via G $\alpha$ i, not the PI3K pathway-linked to the G $\beta \gamma$ subunit. Galanin and GALR1 also inhibit colony formation and tumor growth in vivo. Our results implicate GALR1, a Gi protein-coupled receptor, as a tumor suppressor gene that inhibits cell proliferation via ERK1/2 activation.

Oncogene (2007) 26, 5762-5771; doi:10.1038/sj.onc.1210384; published online 26 March 2007

Keywords: G protein-coupled receptors; mitogen-activated protein kinase; cyclin-dependent kinase inhibitor; head and neck neoplasm

\section{Introduction}

Loss of heterozygosity ( $\mathrm{LOH}$ ) on chromosome $18 \mathrm{q}$ is associated with poor survival in head and neck cancer

Correspondence: Professor TE Carey, Departments of Otolaryngology and Pharmacology, University of Michigan, 1301 East Ann Street, 6020 KHRI, Ann Arbor, MI 48109-0506, USA.

E-mail: careyte@umich.edu

Received 16 August 2006; revised 29 January 2007; accepted 1 February 2007; published online 26 March 2007
(Pearlstein et al., 1998). Galanin receptor 1 (GALR1) (Cody et al., 1997), a G protein-coupled receptor (GPCR), maps to the region of $18 \mathrm{q}$ loss (Takebayashi et al., 2000, 2004). Furthermore, GALR1 is frequently silenced by methylation and deacetylation in head and neck squamous cell carcinomas (HNSCC) (Misawa et al., 2004). Thus, we postulated that abnormalities of GALR1 might have functional consequences in HNSCC.

GPCRs and their ligands regulate multiple cellular functions, and some have been implicated in cancer cell growth, metastasis and apoptosis (Lahlou et al., 2003; Yang et al., 2005). Galanin, is a 30 amino-acid neuropeptide (Tatemoto et al., 1983) that activates all three galanin receptors, GALR1, GALR2 and GALR3 (Berger et al., 2004; Lu et al., 2005). Galanin and its receptors are abundant in the peripheral and central nervous system where they mediate diverse physiological activities (Branchek et al., 2000). Wittau et al. (2000) reported that GALR2 initiates signaling in small-cell lung cancer via $\mathrm{Gq}, \mathrm{Gi}$ and $\mathrm{Gq}_{12}$ proteins. Berger et al. (2004) reported that exogenous expression of GALR2 inhibited cell proliferation and induced apoptosis in neuroblastoma cells, in contrast, GALR1 inhibited proliferation only. Henson et al. (2005) reported that GALR1 has antiproliferative effects in squamous carcinoma cells. However, others have reported that galanin receptors induce mitogenic signaling properties via extracellular-regulated protein kinase (ERK)1/2 (Seufferlein and Rozengurt, 1996). GALR1 was reported to couple to heterotrimeric $\mathrm{G}$ proteins of the $\mathrm{Gi}$ type, which inhibit cAMP and stimulate mitogenactivated protein kinase (MAPK) via $\mathrm{G} \beta \gamma$ (Wang et al., 1998). Thus, the role of galanin and GALR1induced ERK1/2 activation and the mechanisms involved are not well established.

We studied the signaling contribution of GALR 1 in a human oral carcinoma cell line that expresses neither galanin nor GALR1. We showed that GALR1 induces antiproliferation effects by a mechanism involving $\mathrm{G} \alpha \mathrm{i}$ activation of ERK1/2, and consequent regulation of cell cycle control proteins. 


\section{Results}

Exogenous GALR1 expression does not affect GALR2 and GALR3 $m R N A$ expression

UM-SCC-1 cells express message for GALR2, and GALR3 but not GALR1 as determined by qualitative reverse transcriptase-polymerase chain reaction (RTPCR) experiments (Figure 1a). This is consistent with our findings that promoter hypermethylation silences GALR1 in these cells (Misawa et al., 2004). Exogenous GALR1-hemaglutinin (HA) expression in UM-SCC-1GALR1 was assessed by immunoblotting (Figure 1b) and immunofluorescence (Figure 1c). GALR1-HA localized to the cytoplasmic membrane as expected for a GPCR (Figure 1c). As expected, GALR1 expression in UM-SCC-1-GALR1 was significantly increased relative to UM-SCC-1-mock $(P<0.01)$, but no significant differences in GALR2 and GALR3 expression were detected by quantitative RT-PCR (Figure 1d). Thus, differences obtained with UM-SCC-1-GALR1 and UM-SCC-1-mock following galanin stimulation should reflect mainly the function of GALR1.
Galanin and GALR1 induce ERK1/2 activation without $P I 3 K$ pathway stimulation

To determine the GALR1 signaling pathway, we evaluated galanin and GALR1-mediated ERK1/2, protein kinase $\mathrm{B}(\mathrm{Akt})$ and $\mathrm{S} 6$ protein kinase $(\mathrm{S} 6 \mathrm{~K})$ activation. ERK1/2 activation in UM-SCC-1-GALR1 was apparent at $0.3 \mathrm{~h}$, increased to a peak level at $1-3 \mathrm{~h}$ and declined slowly through $24 \mathrm{~h}$ after stimulation (Figure 2a). ERK1/2 phosphorylation intensity was normalized to epidermal growth factor (EGF)-induced ERK1/2 activation and was significantly stronger in UM-SCC-1-GALR1 than in UM-SCC-1-mock cells at all time points, $0.3,12$ and $24 \mathrm{~h}(P<0.05)$ and 1,3 and $6 \mathrm{~h}(P<0.01)$ (Figure $2 \mathrm{a})$. As shown in Figure $2 \mathrm{~b}$, both Akt and pS6K, downstream mediators of phosphatidylinositol 3-kinase (PI3K), were activated at all time points, but there were no significant differences between UM-SCC-1-GALR1 and UM-SCC-1-mock cells (Figure 2b). Thus, GALR1 stimulates ERK1/2 activation but is not required for PI3K signaling in UMSCC-1 cells. To determine if GALR2 influences ERK activation mediated by galanin and GALR1, galanin
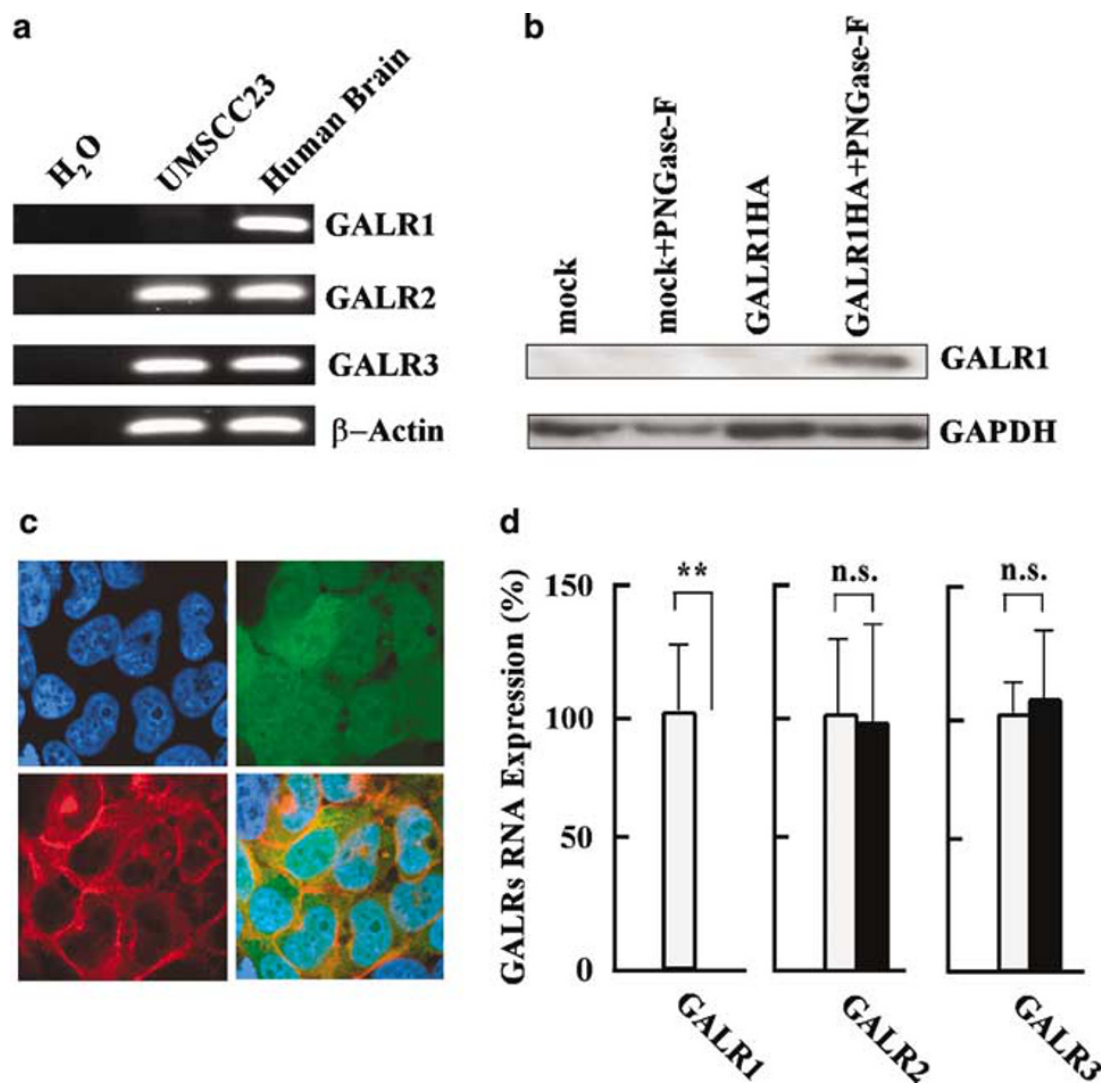

Figure 1 Galanin receptor expression in parental and GALR1HA transfected UM-SCC-1 cells. (a) RT-PCR shows that parental UM-SCC-1 cells express message for GALR2 and GALR3 but not GALR1. (b) Immunoblotting shows exogenous GALR1 expression in pCMVGALR1HAIresGFP-transfected cells detected by antibody to the HA-tag. Lanes labeled mock and GALR1 contain protein lysates from mock transfected and GALR1HA transfected UM-SCC-1 cells. Lanes labeled mock + PNGase and GALR1 + PNGase contain protein from cells lysed, and then digested with $N$-Glycosidase F. (c) Exogenous GALR1 localizes to the cytoplasmic membrane in UM-SCC-1-GALR1HA cells. UM-SCC-1-GALR1 cells were stained with mouse monoclonal anti-HA tag antibody and Hoechst 33342. Photographs show Hoechst 33342 (upper left), GFP (upper right), HA-tag (lower left) and Merge (lower right) (magnification $\times 400$ ). (d) Quantitative RT-PCR for galanin receptor expression in UM-SCC-1-GALR1 transfected (open bar) and UM-SCC-1-mock cells (closed bar). Galanin receptor expression is presented relative to that of $\beta$-actin. Percentages indicate the RNA expression in UM-SCC-1-GALR1 cells relative to UM-SCC-1-mock cells. ${ }^{* *} P<0.01$. 
a
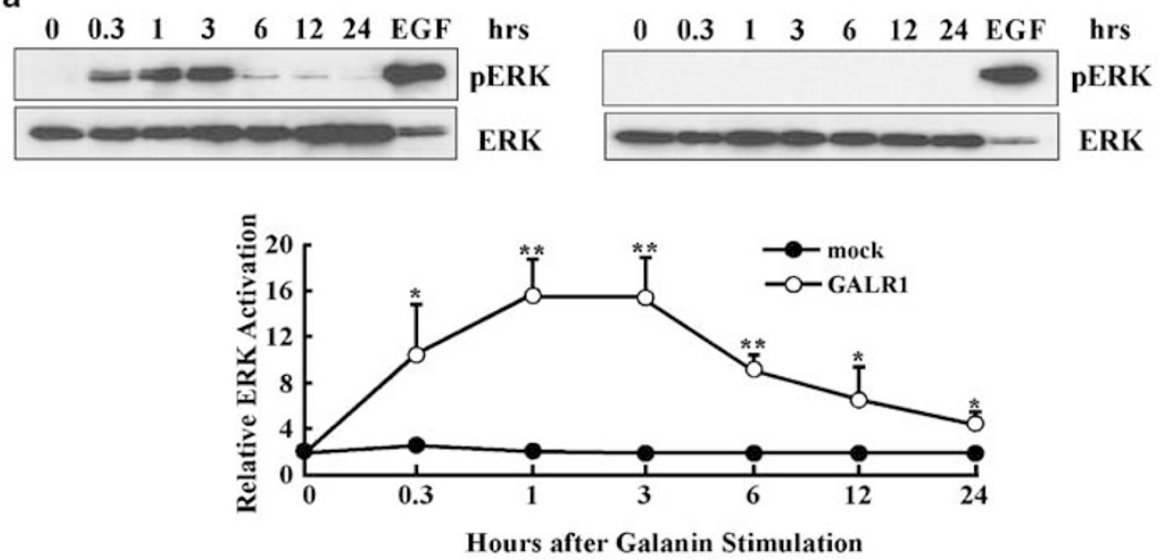

b
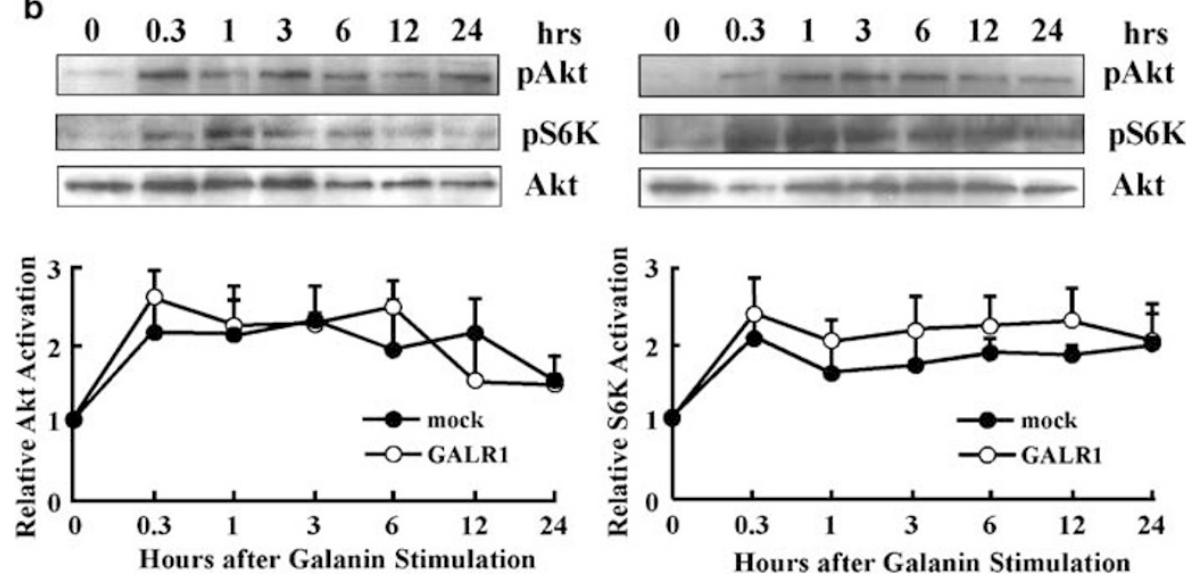

C

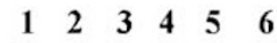

\begin{tabular}{|c|c|c|c|}
\hline & pERK & $\begin{array}{l}\text { 1. mock } \\
\text { 2. mock + Galanin }\end{array}$ & $\begin{array}{l}\text { 4. GALR1 } \\
\text { 5. GALR1 + Galanin }\end{array}$ \\
\hline & RK & 3. mock + Galanin + GALP & 6. GALR1 + Galanin + GALP \\
\hline
\end{tabular}

Figure 2 Effect of galanin stimulation on ERK1/2, Akt and S6K activation in the transfected UM-SCC-1 cells. (a) Immunoblot showing galanin-induced ERK1/2 activation in UM-SCC-1-GALR1 (upper left) and UM-SCC-1-mock cells (upper right). The relative intensity of the stained bands in UM-SCC-1-GALR1 (open circle) and UM-SCC-1-mock (closed circle) cells was assessed by densitometry (center panel). ${ }^{* *} P<0.01,{ }^{*} P<0.05$. (b) Galanin-induced Akt and S6K activation in UM-SCC-1-GALR1 (upper left) and UM-SCC-1-mock cells (upper right). The intensity of Akt bands (lower left) or S6K (lower right) in UM-SCC-1-GALR1 (open circle) and UM-SCC-1-mock (closed circle) cells were assessed by densitometry. (c) Erk activation after simultaneous stimulation with Galanin and GALP.

and a selective GALR2 agonist, GALP, were added simultaneously to UM-SCC-1 and UM-SCC-1-GALR1 cultures. GALP did not alter ERK activation (Figure 2c), indicating that GALR1 is the primary receptor for activation of this pathway.

Galanin and GALR1 inhibit cell proliferation

Figure 3a shows dose- and time-dependent proliferation assays after galanin treatment. As the galanin concentration increased, the cell number at $24 \mathrm{~h}$ decreased in UM-SCC-1-GALR1 but not in UM-SCC-1-mock cells $(P<0.01)$. Furthermore, when the cells were treated with a fixed concentration of galanin $(1 \mu \mathrm{M})$ and counted at 24,48 and $72 \mathrm{~h}$, the difference between UM-SCC-1GALR1 and UM-SCC-1-mock cells was apparent at
$24 \mathrm{~h}$, and increased in a time-dependent manner $(P<0.01)$. To clarify the mechanism of GALR1-induced inhibition of cell proliferation, we examined bromodeoxyuridine (BrdU) incorporation in UM-SCC-1GALR1 and UM-SCC-1-mock cells. Compared with UM-SCC-1-mock cells, UM-SCC-1-GALR1 cells showed a significantly lower rate of BrdU incorporation after galanin treatment $(P<0.01)$ (Figure $3 b)$. Addition of GALP did not alter the galanin-induced inhibition of cell proliferation (Figure 3c).

Galanin and GALR1 downregulate cyclin D1 and upregulate $p 27^{\text {Kip } 1}$ and $p 57^{\text {Kip } 2}$

Tumor-associated alterations in cell proliferation frequently affect cyclin-dependent kinases (Cdks) and their 
a

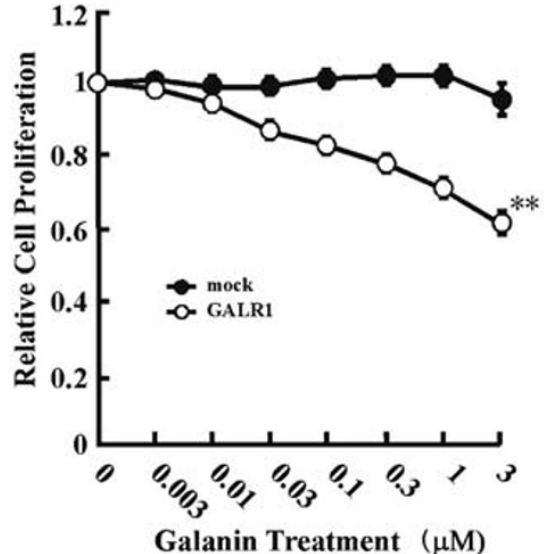

b
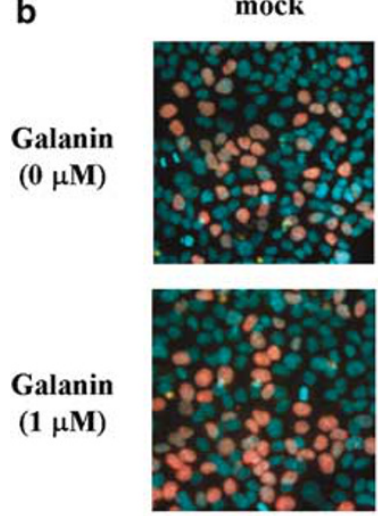

C

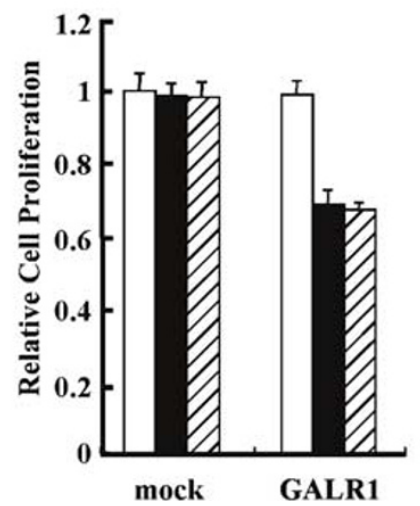

GALR1
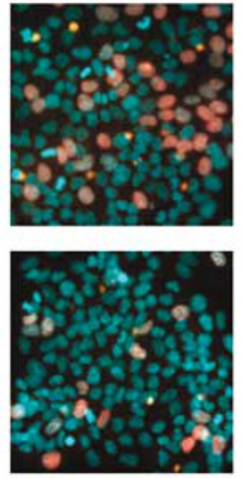

$\square$ no treatment

Galanin

Galanin + GALP

Figure 3 Effect of galanin on growth of GALR1- and mock-transfected UM-SCC-1 cells. (a) Relative cell proliferation as a function of galanin concentration. Cells were cultured with various concentrations of galanin for $24 \mathrm{~h}$ (left) or with $1 \mu \mathrm{M}$ galanin for indicated periods of time (right) ${ }^{* *} P<0.01$. (b) BrdU incorporation after galanin treatment. The BrdU-positive (pink) cells were significantly decreased in galanin treated UM-SCC-1-GALR1 cells (left) (magnification $\times 200$ ). BrdU incorporation rate is the ratio of BrdUpositive cell number to total cell number (right). Closed bars indicate galanin treatment and open bars indicate no treatment (right) $* * P<0.01$. (c) Effect of simultaneous stimulation of galanin and GALP on cell growth.

regulators such as $\mathrm{p} 27^{\mathrm{Kip} 1}, \mathrm{p} 57^{\mathrm{Kip} 2}$ and cyclin D1. As shown in Figure 4a, cyclin D1 expression decreased in galanin-treated UM-SCC-1-GALR1 but not in UMSCC-1-mock cells. Densitometric measurements showed cyclin D1 expression was significantly suppressed $(P<0.01)$. In contrast, $\mathrm{p} 27^{\mathrm{Kip} 1}$ and $\mathrm{p} 57^{\mathrm{Kip} 2}$ expression increased after galanin treatment in UM-SCC-1GALR1, but not in UM-SCC-1-mock cells. Galanin also significantly enhanced both $\mathrm{p} 27^{\mathrm{Kip} 1}$ and $\mathrm{p} 57^{\mathrm{Kip} 2}$ expression $(P<0.01)$ (Figure $4 \mathrm{~b}$ and $\mathrm{c})$. These data suggest that GALR1-induced inhibition of proliferation is mediated by regulation of $\mathrm{p} 27^{\mathrm{Kip} 1}, \mathrm{p} 57^{\mathrm{Kip} 2}$ and cyclin $\mathrm{D} 1$.

Galanin and GALRI induce active ERK1/2 and inhibit cell proliferation via the Gai pathway

As GALR1 activates ERK1/2 and inhibits cell proliferation, we investigated if ERK1/2 activation is 
a
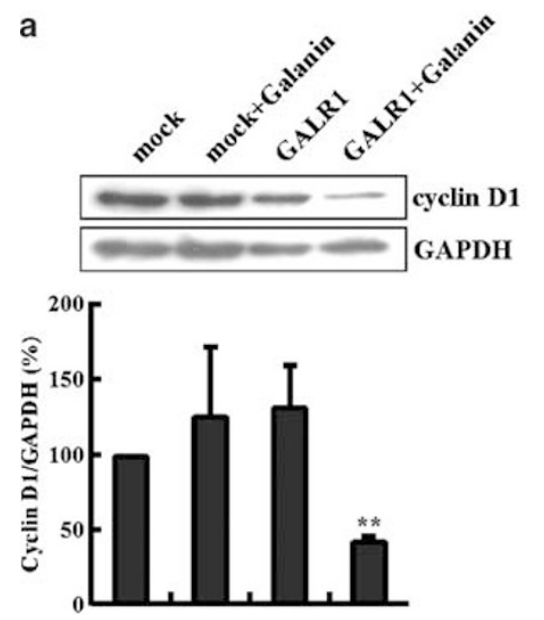

b
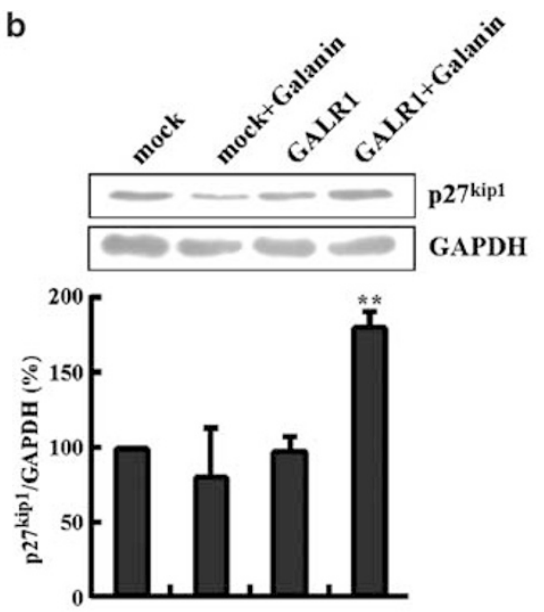

c
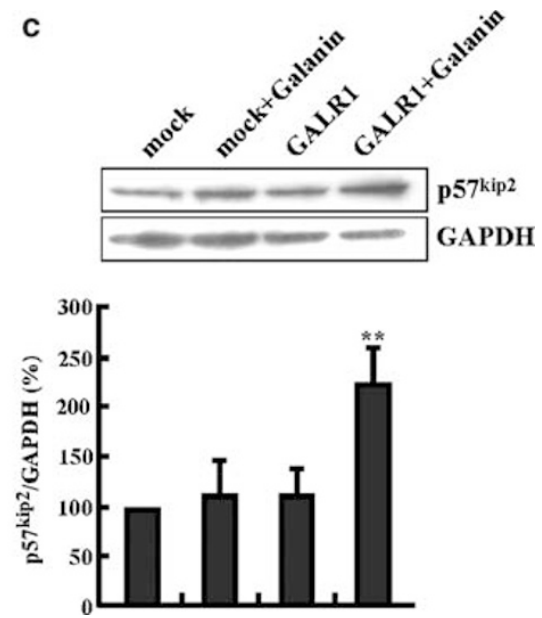

Figure 4 Galanin and GALR1 regulate cell cycle control genes. Serum-starved cells were treated with $1 \mu \mathrm{M}$ galanin for $24 \mathrm{~h}$ as described and expression of cyclin D1 (a), p2 $7^{\mathrm{Kip} 1}(\mathbf{b})$ and p57 $7^{\mathrm{Kip} 2}$ (c) were assessed by immunoblotting. The band intensities were determined by densitometry and normalized to GAPDH. ${ }^{* *} P<0.01$.

required for the inhibition of cell proliferation and determined the pathway used for this effect. U0126 and pertussis toxin (PTX) pretreatment each abrogated galanin- and GALR1-mediated ERK1/2 activation, whereas LY294002 did not, indicating that GALR1induced ERK1/2 activation involves the PTX-sensitive G $\alpha$ i pathway, not the PI3K pathway linked to the G $\beta \gamma$ subunit (Figure 5a). Similarly, U0126 and PTX each blocked galanin-induced GALR1-mediated cell growth inhibition, whereas LY294002 did not (Figure 5b), indicating that cell growth inhibition also involves the PTX-sensitive G $\alpha \mathrm{i}$ pathway. Thus, GALR1-induced ERK1/2 activation and cell growth inhibition are closely related. To verify the relationship between GALR1induced ERK1/2 activation and cell growth inhibition, we assessed cyclin D1, p27 $7^{\mathrm{Kip} 1}$ and $\mathrm{p} 57^{\mathrm{Kip2}}$ expression in galanin-treated UM-SCC-1-GALR1 with or without U0126. As shown in Figure 5c, p27 ${ }^{\text {Kip1 }}$ and p57 Kip2 expression increased significantly after galanin treatment, but this increase was abrogated by U0126 pretreatment. U0126 also abrogated the galanin-induced decrease in cyclin D1 expression. In each case, galanintreated UM-SCC-1-GALR1 exhibited a significant difference in cyclin D1, p $27^{\mathrm{Kip} 1}$ and $\mathrm{p} 57^{\mathrm{Kip} 2}$ expression when U0126-pretreated and untreated cells were compared. Thus, GALR1-mediated ERK1/2 activation, via stimulation of the Gi pathway rather than the PI3K pathway, contributes to regulation of cell cycle control proteins and growth inhibition.

\section{Galanin and GALR1 inhibit colony formation and tumor} growth in vivo

As shown in Figure 6a, transient transfection of the parental UM-SCC-1 cells with pCMVGalanin resulted in galanin secretion $(7.2 \mathrm{ng} / \mathrm{ml})$ that is significantly higher than untransfected $(0.16 \mathrm{ng} / \mathrm{ml})$ or mock-transfected cells $(0.19 \mathrm{ng} / \mathrm{ml}) \quad(P<0.01)$. pCMVGalanin transfection significantly suppressed colony formation in UM-SCC-1-GALR1 cells (124.1 versus 51.4)
$(P<0.01)$. In UM-SCC-1-mock cells, pCMVGalanin or mock-transfection did not significantly affect colony formation (132.6 versus 168.4) (Figure 6b). To assess the effect of GALR1 on in vivo tumor formation and growth, UM-SCC-1-GALR1 or UM-SCC-1-mock cells with or without pCMVGalanin transfection were injected subcutaneously (s.c.) into nude mice. Group 1 received UM-SCC-1-mock cells transfected with the pCMV empty vector. Group 2 received UM-SCC-1GALR1 cells transfected with the pCMV empty vector. Group 3 received UM-SCC-1-mock cells transfected with pCMVGalanin and Group 4 received UM-SCC-1GALR1 cells transfected with pCMVGalanin. As shown in Figure 6c, after s.c. injection, tumor formation rates of Group $1(80 \%), 2(70 \%)$ and $3(70 \%)$ were significantly higher than Group $4(30 \%)(P<0.05)$. In addition, the three tumors in Group 4 that did become established grew more slowly than those in the other three groups $(P<0.05)$.

\section{Discussion}

Our previous work suggested that GALRl might function as a tumor suppressor gene (Takebayashi et al., 2004; Henson et al., 2005). Consistent with this hypothesis, the present study shows that GALR1 signaling can inhibit cell proliferation by a mechanism dependent on ERK1/2 activation. Furthermore, ERK1/ 2 activation leads to induction of $\mathrm{p} 27^{\mathrm{Kip} 1}, \mathrm{p} 57^{\mathrm{Kip} 2}$ and to suppression of cyclin D1.

GALR1 is a classical Gi-coupled receptor (Wang et al., 1998), and galanin is known to stimulate ERK1/2 activation (Seufferlein and Rozengurt, 1996). Generally, the G $\alpha$ i pathway activates ERK $1 / 2$ by membrane recruitment of rap1GAP II and reduction of GTPbound Rap1 (Mochizuki et al., 1999). Henson et al. (2005) observed that a blocking antibody to GALR1 activated ERK and inhibited growth in laryngeal cancer 


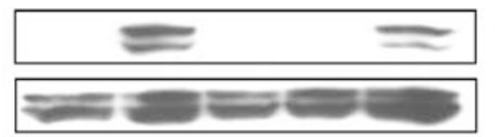

pERK
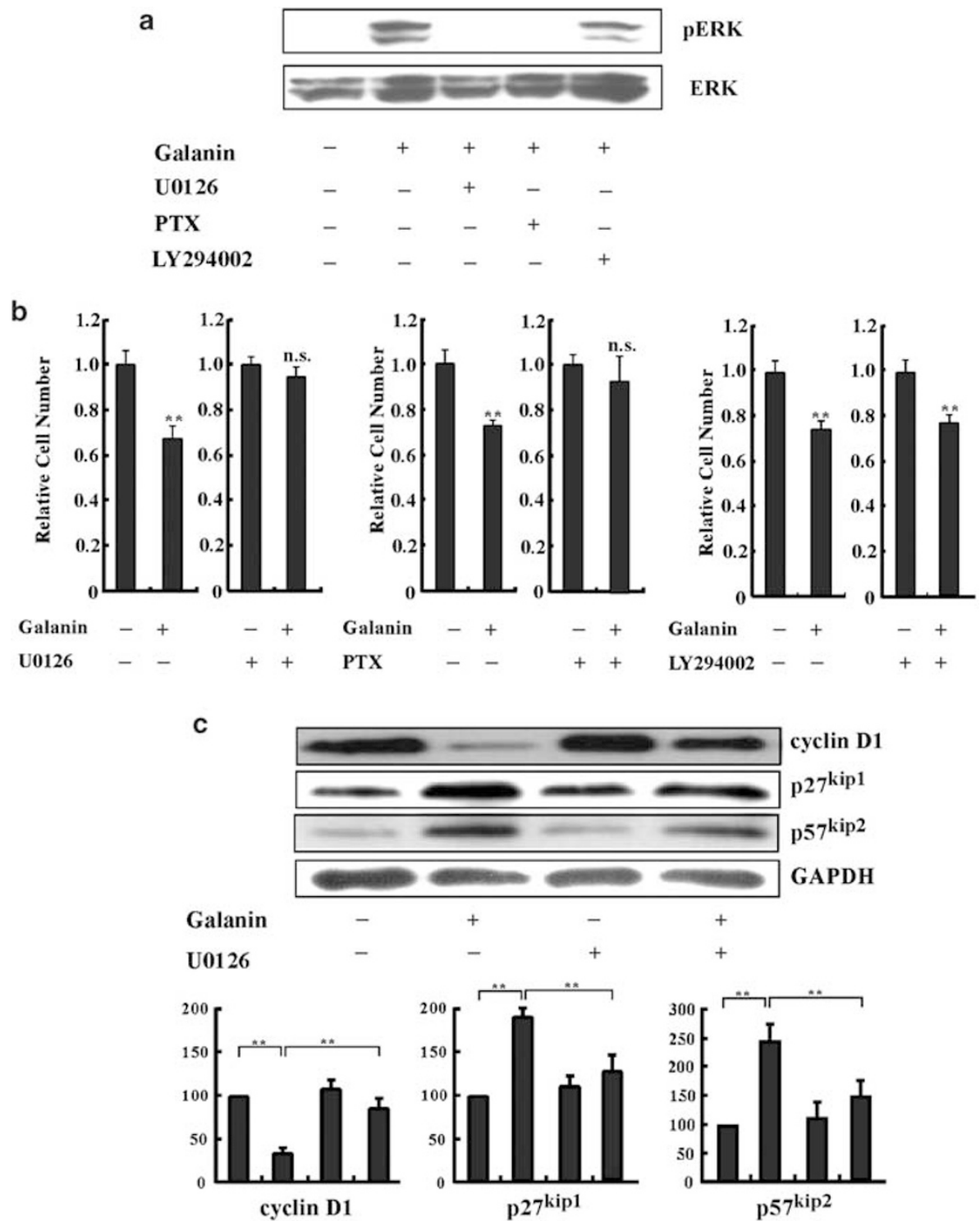

Figure 5 The G $\alpha$ i pathway leads to ERK1/2 activation, cell growth inhibition and cell cycle control gene regulation. (a) Serumstarved UM-SCC-1-GALR1 cells were pretreated with U0126, PTX or LY294002 before galanin treatment, and evaluated for ERK1/2 phosphorylation. (b) Effect of galanin and signaling pathway inhibitors on cell proliferation. Serum-starved UM-SCC-1-GALR1 cells were pretreated with inhibitors before galanin treatment. Twenty-four hours later, the cells were counted $* * P<0.01$. (c) Effects of galanin and ERK1/2 inhibition on cell cycle regulator proteins. Serum-starved UM-SCC-1-GALR1 cells were pretreated with U0126 before galanin treatment. Expression of cyclin D1, p2 $7^{\mathrm{Kip} 1}$ and $\mathrm{p} 57^{\mathrm{Kip} 2}$ were assessed as described in 'Materials and methods'. $* * P<0.01$.

cells. It was not clear in that study if GALR1 signaling activates or inhibits ERK $1 / 2$. In the present study, we demonstrated that galanin and GALR1-mediate PTXsensitive ERK1/2 activation using UM-SCC-1-GALR1 cells that express no endogenous GALR1.

At one level, it is counterintuitive that activation of ERK1/2 by GALR1 could lead to an antiproliferative phenotype. However, antiproliferative effects consequent to ERK1/2 activation are known. Ferbeyre et al. (2002) showed that Ras could lead to cell-cycle arrest or cell death owing to activation of the MAPK/ERK kinase (MEK)/ERK pathway. Moreover, the small molecule UCN-01 induces cell cycle arrest by activation of the MEK/ERK pathway (Facchinetti et al., 2004). Peptide-induced stimulation of ERK1/2 via other GPCRs including somatostatin type 2 (Lahlou et al., 2003), $\beta 1$ bradykinin (Dixon et al., 2002) and angiotensin II receptors (Otis et al., 2005) has also been linked to inhibition of cell proliferation. Thus, the galanin/ GALR1-induced antiproliferative effect consequent to ERK $1 / 2$ activation is not unprecedented. GALR 1 has $30-34 \%$ protein homology with the somatostatin receptors (Branchek et al., 2000) and the latter inhibits cell proliferation through ERK2 activation (Lahlou et al., 2003). The current study shows that galanin- and GALR1-induced growth inhibition is blocked by an 


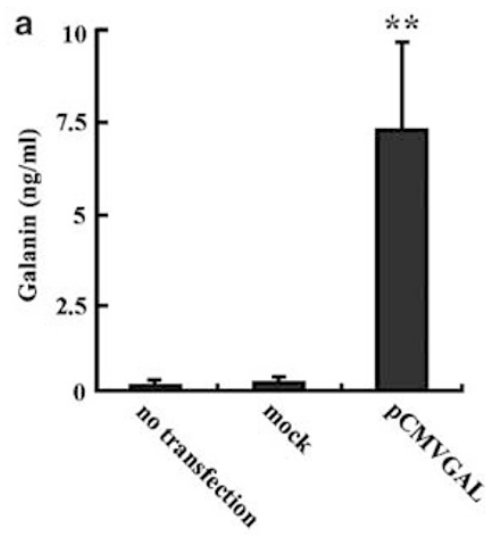

b
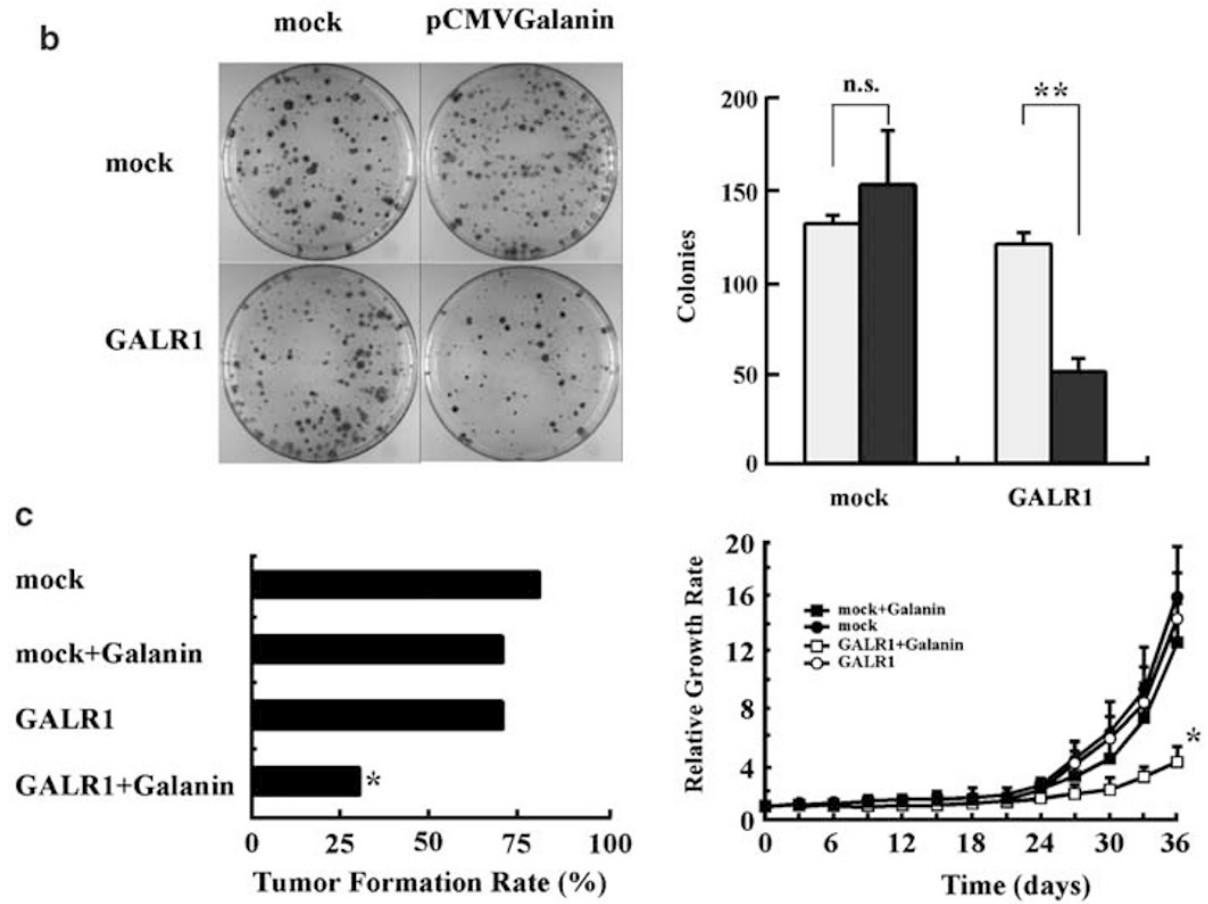

Figure 6 Galanin and GALR1 inhibit colony formation in vitro and tumor growth in vivo. (a) pCMVGalanin-transfected cells secrete galanin. Galanin in the media of UM-SCC-1 cells transfected with pCMVGalanin was measured with a galanin-specific ELISA as described in methods $(* * P<0.01)$. (b) Inhibition of colony formation by galanin and GALR1. Clonogenic survival for Hygromycin B selected cells was determined by crystal violet staining (left). The colony formation potential was determined though three independent experiments (right). Closed bars indicate pCMVgalanin transfection. Open bars indicate mock-transfection ** $P<0.01$. (c) Galanin and GALR1 inhibit tumor growth in vivo. Tumor formation rates are the percentages of tumors at 4 weeks (left). The relative tumor volume was calculated as described in 'Materials and methods' (right). ${ }^{*} P<0.05$.

ERK1/2-specific inhibitor and thus is dependent on ERK1/2 activation. The ultimate cellular response (i.e., growth inhibition versus proliferation) via the ERK1/2 pathway depends on the strength and duration of activation (Pumiglia and Decker, 1997). Transient or modest activation may contribute to cell cycle progression, whereas sustained high levels and prolonged activation may result in growth arrest (Pumiglia and Decker, 1997; Tsukada et al., 2001; Dixon et al., 2002; Han et al., 2005). The small GTP-binding proteins might be also important to determine the ultimate cellular response by ERK1/2 activation (Lahlou et al., 2003). Woods et al. (1997) showed that expression of cyclin-dependent kinase inhibitors (CKI) such as p21 ${ }^{\text {Cip1 }}$ versus activation of mitogenesis depends on the level of Ras activation. Modest levels of Ras activation stimulated mitogenesis, but higher levels led to an increase in CKI expression and mitogenic inhibition (Woods et al., 1997). More recently, another Ras family member, Rap 1 and its downstream effector B-Raf, were linked to ERK1/2 activation and consequent cell growth arrest and/or differentiation through a Ras-independent mechanism (Gendron et al., 2003; Lahlou et al., 2003). Our data demonstrate that galanin and GALR1-mediated ERK1/2 activation increased 15-fold up to $3 \mathrm{~h}$ and remained above basal levels for $24 \mathrm{~h}$. Lahlou et al. (2003) showed that the cellular decision to induce $\mathrm{p} 27^{\mathrm{Kip} 1}$ and $\mathrm{G}_{1}$ cell cycle arrest is dictated by the cumulating effect of 
ERK1/2-dependent and ERK1/2-independent mitogenic effects. These findings support our data that galanin and GALR1 induce growth inhibition through ERK $1 / 2$ activation. In this study, galanin also stimulated the PI3K pathway in both UM-SCC-1-GALR1 and UM-SCC-1-mock, indicating that GALR2 or GALR3 mediates that effect. It is possible that heterodimers of the galanin receptors mediate the galanin effects we observed, however, addition of galaninlike peptide (GALP) did not affect ERK activation or cell growth inhibition (Figures $2 \mathrm{c}$ and $3 \mathrm{c}$ ), suggesting that GALR1 is the primary mediator of this pathway.

The ability of $\mathrm{G} \alpha \mathrm{i}$ protein to activate the ERK1/2 pathway is well documented (Mochizuki et al., 1999). Although both the Gai- and the $\mathrm{G} \beta \gamma$-dependent pathways can activate ERK1/2, the mechanism by which the ERK1/2 pathway can lead to inhibition of cell proliferation is not fully understood. Wang et al. (1998) showed that GALR1 couples to $\mathrm{Gi}$ but induces activation of MAPK activity via $\mathrm{G} \beta \gamma$ protein in Chinese hamster ovary cell line $(\mathrm{CHO})$ cells. In our study, the involvement of Gai protein in GALR1-mediated ERK1/ 2 activation is based on the sensitivity of galanin- and GALR1-mediated ERK1/2 activation to PTX. PI3K can contribute to GPCR-mediated ERK1/2 activation by a mechanism involving $\mathrm{G} \beta \gamma$ subunits (Kranenburg and Moolenaar, 2001). In our data, the PI3K inhibitor LY294002 did not prevent either ERK1/2 activation or cell growth suppression induced by galanin and GALR1. The results indicate that GALR1 inhibits proliferation consequent to ERK1/2 activation via the G $\alpha$ i pathway, which is closely related to cell cycle arrest.

Furthermore, galanin and GALR1 regulated the expression of $\mathrm{p} 27^{\mathrm{Kip} 1}, \mathrm{p} 57^{\mathrm{Kip} 2}$ and cyclin D1, and these effects were blocked by U0126 (Figure 5c). p27 $7^{\text {Kipl }}$ and $p 57^{\text {Kip } 2}$ are known as a tumor suppressor genes, and p27 ${ }^{\mathrm{Kip} 1}$ levels are associated with prognosis in many different tumors, including non-small-cell lung cell carcinoma, gastric carcinoma and laryngeal carcinoma (Esposito et al., 1997; Masuda et al., 2002; Hoffmann et al., 2005; Massarelli et al., 2005). Overexpression of cyclin D1 occurs at a high frequency in a variety of carcinomas including those of breast, pancreas, and head and neck, and is associated with poor prognosis (Kong et al., 2000; Akervall et al., 2003). The fact that galanin and GALR1 can regulate these genes suggests that GALRI is a tumor suppressor in head and neck cancer cells.

Galanin and GALR1 also inhibit cell proliferation and suppress colony-forming potential. The suppression of colony formation might result from both growth inhibition and blockade of anchorage-independent survival by GALR1. As with the in vitro findings, tumor cells expressing both galanin and GALR1 have a reduced in vivo tumor formation rate and significantly decreased tumor growth in vivo.

In conclusion, we have shown that galanin and GALR1 induce a marked and prolonged ERK1/2 activation, upregulation of $\mathrm{p} 27^{\mathrm{Kip} 1}$ and $\mathrm{p} 57^{\mathrm{Kip} 2}$, downregulation of cyclin D1, and consequent inhibition of cell proliferation. These data support the concept that $G A L R 1$ is a likely tumor suppressor in head and neck cancer cells.

\section{Materials and methods}

\section{Cell culture and proliferation assay}

UM-SCC-1, established from a human oral carcinoma, was cultured in Dulbecco's modified Eagle's Medium (Gibco, Grand Island, NY, USA) supplemented with 10\% heatinactivated fetal bovine serum. The GALR1 sequence was obtained from a human cDNA library (Invitrogen, Carlsbad, CA, USA), C-terminal HA-tagged, and subcloned into the pcDNA3 vector (Invitrogen) containing an internal ribosomal entry site (Ires) and green fluorescent protein (GFP) sequence. The pCMVIresGFP vector was used as a transfection control. The UM-SCC-1-GALR1 and UM-SCC-1-mock cells were transfected with pCMVGALR1HAIresGFP or pCMVIresGFP, respectively, using lipofectamine (Invitrogen), and selected for GFP-positive cells. Twenty-four hours after plating, stably transfected cells were fed with serum-free medium (SFM) containing $0.1 \%$ bovine serum albumin for $24 \mathrm{~h}$ to induce quiescence. Then, the galanin (ANASPEC, San Jose, CA, USA) and in some experiments $1 \mu \mathrm{M}$ (GALP) (Bachem Feinchemikalien AG, Bubendorf, Switzerland) were added. Activity of GALP was determined using GALR2transfected UM-SCC-1 cells (data not shown). The ERK1/2 inhibitor U0126 or PI3K inhibitor LY294002 (Cell Signal Technology, Beverly, MA, USA) was added $1 \mathrm{~h}$ before galanin treatment. PTX (Sigma, St Louis, MO, USA) was added $10 \mathrm{~h}$ before treatment. Cell proliferation was measured by counting cells with a Coulter counter model Z1 (Beckman Coulter Inc., Hialeah, FL, USA). For the BrdU incorporation assay, the cells were incubated with $10 \mu \mathrm{M}$ BrdU (Caltag Laboratories, Burlingame, CA, USA), and stained with mouse monoclonal anti-BrdU antibody (Caltag Laboratories) followed by Alexa Fluor 546 goat anti-mouse $\mathrm{IgG}_{1}$ (Molecular Probes, Leiden, The Netherlands).

\section{Qualitative and quantitative $R T-P C R$}

Total RNA was isolated using RNeasy mini kit (Qiagen Inc., Yalencia, CA, USA). Complementary DNA (cDNA) was synthesized according to the manufacturer's instructions. GALRs and $\beta$-actin were amplified using FastStart Taq DNA Polymerase (Roche Diagnostics GmbH, Penzberg, Germany) for Qualitative and LightCycler FastStart DNA Master SYBR Green I (Roche Diagnostics $\mathrm{GmbH}$ ) for quantitative RT-PCR. Primers were designed as follows: GALR1 forward, 5'-ctgcttctgctatgccaaggtc; reverse 5'-agga gattccaaacaccacaacc, GALR2 forward, 5'-agcatcctgacggtt gatgtgg; reverse, 5'-ccccaacaatgactccaactc, GALR3 forward, $5^{\prime}$-ctgatgcccagaacatttcactg, reverse, $5^{\prime}$-tgtgcccagcaggaagattagg. $\beta$-Actin forward, $5^{\prime}$-actatcggcaatgagcggtt, reverse, $5^{\prime}$-tgccacag gattccatacce.

\section{Immunoblotting}

Cells were lysed with $0.1 \%$ Nonidet-P 40 lysis buffer containing protease (Calbiochem, La Jolla CA, USA) and phosphatase inhibitor cocktails (Sigma). Equal amounts of protein were electrophoresed on $10 \%$ sodium dodecyl sulfate-polyacrylamide gel electrophoresis gels and transferred to Hybond-P (Amersham Biosciences, Buckinghamshire, UK). The membranes were incubated overnight with primary antibody at $4{ }^{\circ} \mathrm{C}$, followed by incubation with anti-mouse or anti-rabbit secondary antibody horseradish peroxidase conjugate (Amersham 
Biosciences). Densitometric measurements of the bands were done using ChemiImager 4400 (Alpha Innotech, San Leandro, CA, USA). To avoid protein aggregation, cells lysates were treated with $1000 \mathrm{U}$ of $N$-Glycosidase $\mathrm{F}$ (New England BioLabs, Beverly, MA, USA), and subjected to electrophoresis without boiling. The mouse monoclonal anti-HA tag antibody (Convance, Berkeley, CA, USA) was used as primary antibody to detect exogenous GALR1. ERK1/2, Akt and p70 S6K activation were evaluated with rabbit polyclonal phosphoERK1/2, Akt or S6K antibody and total ERK1/2 or Akt antibody (Cell Signaling Technology). The expression of $\mathrm{p} 27^{\mathrm{Kip} 1}$, p57 $7^{\mathrm{Kip} 2}$ and cyclin D1 were detected with murine monoclonal antibodies for p27 ${ }^{\mathrm{Kip} 1}$, p57 $7^{\mathrm{Kip} 2}$ (Lab Vision, Fremont, CA, USA) and cyclin D1 (DakoCytomation Norden A/S, Glostrup, Denmark). Glyceraldehyde-3-phosphate dehydrogenase (GAPDH) was detected by mouse monoclonal antiGAPDH (Chemico International, Temecula, CA, USA) as an internal control for protein loading.

\section{Immunocytochemistry}

Cells were grown on coverslips for $24 \mathrm{~h}$, fixed with $4 \%$ paraformaldehyde and stained with mouse monoclonal antiHA tag antibody and Hoechst 33342 (Molecular Probes). Primary antibody binding was detected with Alexa Fluor 546 goat anti-mouse $\mathrm{IgG}_{1}$ (Molecular Probes). The localization of exogenous GALR1 was determined using the Olympus FV500 Confocal Microscope (Olympus Corporation, Tokyo, Japan).

\section{Colony formation assay}

A human galanin expression vector, pCMVGalanin, was constructed by subcloning the galanin precursor sequence, preprogalanin, obtained from human brain cDNA library. To confirm that the vector-induced galanin expression, UM-SCC1 cells were transfected with pCMVGalanin and assessed for expression. Twenty-four hours after transfection, the cells were fed with SFM, and $24 \mathrm{~h}$ later, galanin secretion was measured by a human galanin-specific immunoassay kit (Peninsula

\section{References}

Akervall J, Bockmuhl U, Petersen I, Yang K, Carey TE, Kurnit DM. (2003). The gene ratios c-MYC:cyclin-dependent kinase $(\mathrm{CDK}) \mathrm{N} 2 \mathrm{~A}$ and CCND1:CDKN2A correlate with poor prognosis in squamous cell carcinoma of the head and neck. Clin Cancer Res 9: 1750-1755.

Berger A, Lang R, Moritz K, Santic R, Hermann A, Sperl W et al. (2004). Galanin receptor subtype GalR2 mediates apoptosis in SH-SY5Y neuroblastoma cells. Endocrinology 145: $500-507$.

Branchek TA, Smith KE, Gerald C, Walker MW. (2000). Galanin receptor subtypes. Trends Pharmacol Sci 21: 109-117.

Cody JD, Hale DE, Brkanac Z, Kaye CI, Leach RJ. (1997). Growth hormone insufficiency associated with haploinsufficiency at 18q23. Am J Med Genet 71: 420-425.

Dixon BS, Evanoff D, Fang WB, Dennis MJ. (2002). Bradykinin B1 receptor blocks PDGF-induced mitogenesis by prolonging ERK activation and increasing p27Kip1. Am J Physiol Cell Physiol 283: C193-C203.

Esposito V, Baldi A, De Luca A, Groger AM, Loda M, Giordano GG et al. (1997). Prognostic role of the cyclindependent kinase inhibitor p27 in non-small cell lung cancer. Cancer Res 57: 3381-3385.

Facchinetti MM, De Siervi A, Toskos D, Senderowicz AM. (2004). UCN-01-induced cell cycle arrest requires the
Laboratories Inc, San Carlos, CA, USA). pCMVGalanin and a plasmid harboring the Hygromycin $\mathrm{B}$ resistance gene were cotransfected into UM-SCC-1-GALR1 or UM-SCC-1mock cells cultured on $0.8 \%$ agarose-coated dishes. After the selection by Hygromycin B (Invitrogen), clonogenic survival was determined by crystal violet staining.

\section{Tumor xenografts}

To establish cell lines for animal experiments, the pCMVGalanin or empty vector were transfected into UM-SCC-1GALR1 or UM-SCC-1-mock cells, followed by selection in Hygromycin B. Female BALB/c nude mice between the ages of 6-8 weeks (Charles River Laboratories Inc., Wilmington, MA, USA) were given s.c. injections of $5 \times 10^{6}$ cells with $40 \%$ Matrigel (Collaborative Research, Bedford, MA, USA) into each flank. Tumor formation rates were determined as the percentages of persisting tumors of at least $1 \mathrm{~mm}^{3}$ tumor volume after 4 weeks. Tumor size was measured periodically and volume calculated as $0.5 \times L \times W^{2}$, where $L$ is the length $(\mathrm{mm})$ and $W$ is the width $(\mathrm{mm})$. The relative tumor volume was calculated as the ratio of the growing tumor volume at any time to that of the minimum measured size of that tumor.

\section{Statistical analysis}

Results were tested for statistical significance using two-way analysis of variance or Student's $t$-test.

\section{Acknowledgements}

This work was supported by National Institutes of Health grants R01 DE12477, University of Michigan Head and Neck SPORE Grant P50 CA97248, University of Michigan's Cancer Center Support Grant P30 CA46592, a Research Resources Core grant NIH NIDCD P30 DC05188, and a Grant-in-Aid for Scientific Research (No. 452662) from the Ministry of Education, Culture, Sports, Science, and Technology of Japan. transcriptional induction of $\mathrm{p} 21$ (wafl/cip1) by activation of mitogen-activated protein/extracellular signal-regulated kinase kinase/extracellular signal-regulated kinase pathway. Cancer Res 64: 3629-3637.

Ferbeyre G, de Stanchina E, Lin AW, Querido E, McCurrach ME, Hannon GJ et al. (2002). Oncogenic ras and p53 cooperate to induce cellular senescence. Mol Cell Biol 22: 3497-3508.

Gendron L, Oligny JF, Payet MD, Gallo-Payet N. (2003). Cyclic AMP-independent involvement of Rap1/B-Raf in the angiotensin II AT2 receptor signaling pathway in NG108-15 cells. J Biol Chem 278: 3606-3614.

Han J, Tsukada Y, Hara E, Kitamura N, Tanaka T. (2005). Hepatocyte growth factor induces redistribution of p21(CIP1) and p27(KIP1) through ERK-dependent p16(INK4a) up-regulation, leading to cell cycle arrest at G1 in HepG2 hepatoma cells. $J$ Biol Chem 280: 31548-31556.

Henson BS, Neubig RR, Jang I, Ogawa T, Zhang Z, Carey TE et al. (2005). Galanin receptor 1 has anti-proliferative effects in oral squamous cell carcinoma. J Biol Chem 280: 22564-22571.

Hoffmann MJ, Florl AR, Seifert HH, Schulz WA. (2005). Multiple mechanisms downregulate CDKN1C in human bladder cancer. Int $J$ Cancer 114: 406-413. 
Kong S, Amos CI, Luthra R, Lynch PM, Levin B, Frazier ML. (2000). Effects of cyclin D1 polymorphism on age of onset of hereditary nonpolyposis colorectal cancer. Cancer Res 60: 249-252.

Kranenburg O, Moolenaar WH. (2001). Ras-MAP kinase signaling by lysophosphatidic acid and other $\mathrm{G}$ proteincoupled receptor agonists. Oncogene 20: 1540-1546.

Lahlou H, Saint-Laurent N, Esteve JP, Eychene A, Pradayrol L, Pyronnet S et al. (2003). sst2 Somatostatin receptor inhibits cell proliferation through Ras-, Rap1-, and B-Raf-dependent ERK2 activation. J Biol Chem 278: 39356-39371.

Lu X, Lundstrom L, Bartfai T. (2005). Galanin (2-11) binds to GalR3 in transfected cell lines: limitations for pharmacological definition of receptor subtypes. Neuropeptides 39: 165-167.

Massarelli E, Brown E, Tran NK, Liu DD, Izzo JG, Lee JJ et al. (2005). Loss of E-cadherin and p27 expression is associated with head and neck squamous tumorigenesis. Cancer 103: 952-959.

Masuda TA, Inoue $\mathrm{H}$, Sonoda $\mathrm{H}$, Mine $\mathrm{S}$, Yoshikawa $\mathrm{Y}$, Nakayama K et al. (2002). Clinical and biological significance of S-phase kinase-associated protein 2 (Skp2) gene expression in gastric carcinoma: modulation of malignant phenotype by Skp2 overexpression, possibly via p27 proteolysis. Cancer Res 62: 3819-3825.

Misawa K, Ueda Y, Kanazawa T, Misawa Y, Takebayashi S, Mineta $\mathrm{H}$ et al. (2004). CpG hypermethylation of the promoter region and inactivation of GALR 1 in head and neck cancer. Proc Am Assoc Cancer Res 44: 4271.

Mochizuki N, Ohba Y, Kiyokawa E, Kurata T, Murakami T, Ozaki $\mathrm{T}$ et al. (1999). Activation of the ERK/MAPK pathway by an isoform of rap1GAP associated with G alpha(i). Nature 400: 891-894.

Otis M, Campbell S, Payet MD, Gallo-Payet N. (2005). Angiotensin II stimulates protein synthesis and inhibits proliferation in primary cultures of rat adrenal glomerulosa cells. Endocrinology 146: 633-642.

Pearlstein RP, Benninger MS, Carey TE, Zarbo RJ, Torres FX, Rybicki BA et al. (1998). Loss of 18q predicts poor survival of patients with squamous cell carcinoma of the head and neck. Genes Chromosomes Cancer 21: 333-339.
Pumiglia KM, Decker SJ. (1997). Cell cycle arrest mediated by the $\mathrm{MEK} /$ mitogen-activated protein kinase pathway. Proc Natl Acad Sci USA 94: 448-452.

Seufferlein T, Rozengurt E. (1996). Galanin, neurotensin, and phorbol esters rapidly stimulate activation of mitogenactivated protein kinase in small cell lung cancer cells. Cancer Res 56: 5758-5764.

Takebayashi S, Hickson A, Ogawa T, Jung KY, Mineta H, Ueda Y et al. (2004). Loss of chromosome arm 18q with tumor progression in head and neck squamous cancer. Genes Chromosomes Cancer 41: 145-154.

Takebayashi S, Ogawa T, Jung KY, Muallem A, Mineta H, Fisher SG et al. (2000). Identification of new minimally lost regions on $18 \mathrm{q}$ in head and neck squamous cell carcinoma. Cancer Res 60: 3397-3403.

Tatemoto K, Rokaeus A, Jornvall H, McDonald TJ, Mutt V. (1983). Galanin - a novel biologically active peptide from porcine intestine. FEBS Lett 164: 124-128.

Tsukada Y, Miyazawa K, Kitamura N. (2001). High intensity ERK signal mediates hepatocyte growth factorinduced proliferation inhibition of the human hepatocellular carcinoma cell line HepG2. J Biol Chem 276: 40968-40976.

Wang S, Hashemi T, Fried S, Clemmons AL, Hawes BE. (1998). Differential intracellular signaling of the GalR1 and GalR2 galanin receptor subtypes. Biochemistry 37: 6711-6717.

Wittau N, Grosse R, Kalkbrenner F, Gohla A, Schultz G, Gudermann T. (2000). The galanin receptor type 2 initiates multiple signaling pathways in small cell lung cancer cells by coupling to $\mathrm{G}(\mathrm{q}), \mathrm{G}(\mathrm{i})$ and $\mathrm{G}(12)$ proteins. Oncogene 19: 4199-4209.

Woods D, Parry D, Cherwinski H, Bosch E, Lees E, McMahon M. (1997). Raf-induced proliferation or cell cycle arrest is determined by the level of Raf activity with arrest mediated by p21Cip1. Mol Cell Biol 17: 5598-5611.

Yang M, Zhong WW, Srivastava N, Slavin A, Yang J, Hoey T et al. (2005). G protein-coupled lysophosphatidic acid receptors stimulate proliferation of colon cancer cells through the beta-catenin pathway. Proc Natl Acad Sci USA 102: 6027-6032. 